\title{
Mutations in Epigenetic Modifiers in Myeloid Malignancies and the Prospect of Novel Epigenetic-Targeted Therapy
}

\author{
Amir T. Fathi ${ }^{1}$ and Omar Abdel-Wahab ${ }^{2}$ \\ ${ }^{1}$ Department of Medicine, Massachusetts General Hospital, Boston, MA 02114, USA \\ ${ }^{2}$ Human Oncology and Pathogenesis Program and Leukemia Service, Memorial Sloan-Kettering Cancer Center, \\ New York, NY 10065, USA
}

Correspondence should be addressed to Omar Abdel-Wahab, abdelwao@mskcc.org

Received 6 May 2011; Accepted 2 June 2011

Academic Editor: Kevin D. Bunting

Copyright (C) 2012 A. T. Fathi and O. Abdel-Wahab. This is an open access article distributed under the Creative Commons Attribution License, which permits unrestricted use, distribution, and reproduction in any medium, provided the original work is properly cited.

In the recent years, the discovery of a series of mutations in patients with myeloid malignancies has provided insight into the pathogenesis of myelodysplastic syndromes (MDSs), myeloproliferative neoplasms (MPNs), and acute myeloid leukemia (AML). Among these alterations have been mutations in genes, such as IDH1/2, TET2, DNMT3A, and EZH2, which appear to affect DNA and/or histone lysine methylation. Large clinical correlative studies are beginning to decipher the clinical importance, prevalence, and potential prognostic significance of these mutations. Additionally, burgeoning insight into the role of epigenetics in the pathogenesis of myeloid malignancies has prompted increased interest in development of novel therapies which target DNA and histone posttranslational modifications. DNA demethylating agents have been demonstrated to be clinically active in a subset of patients with MDS and AML and are used extensively. However, newer, more specific agents which alter DNA and histone modification are under preclinical study and development and are likely to expand our therapeutic options for these diseases in the near future. Here, we review the current understanding of the clinical importance of these newly discovered mutations in AML and MDS patients. We also discuss exciting developments in DNA methyltransferase inhibitor strategies and the prospect of novel histone lysine methyltransferase inhibitors.

\section{Introduction}

The increasing use of systematic genome-wide discovery efforts in patients with a variety of myeloid malignancies has led to the rapid discovery of a series of recurrent genetic abnormalities underlying these disorders. Remarkably, a large number of these alterations appear to be in genes whose function is known, or suspected, to be involved in epigenetic regulation of gene transcription. In the last 3 years, alone mutations in the genes TET2, IDH1, IDH2, DNMT3a, and EZH2 have all been found in patients with myeloproliferative neoplasms (MPNs), myelodysplastic syndromes (MDSs), and/or acute myeloid leukemia (AML). Although the functional implications of these mutations and how precisely they contribute to abnormal hematopoiesis and leukemogenesis is being heavily investigated and not yet clarified, a number of potentially clinically important implications of these mutations may already be apparent. First, mutations in several of these genes likely hold prognostic importance for patients, and these genetic alterations, thereby, may serve as prognostic markers for risk stratification and aid in therapeutic decision making. Secondly, mutations in several of these genes may specifically impact DNA methylation and/or histone posttranslational modifications in such a manner that is therapeutically targetable. Mutations in several of these genes, such as IDH1 and $\mathrm{IDH} 2$, have been proven to result in a gain-of-enzymatic function which holds the prospect for development of novel targeted therapeutics. This review focuses on the clinical relevance of recently discovered epigenetic alterations in 
patients with myeloid malignancies and the prospect for novel targeted therapeutics against aberrant epigenomic characteristics in patients with MDS and AML.

\section{Recently Identified Mutations in Epigenetic Modifiers in Myeloid Malignancies}

2.1. IDH1 and IDH2 Mutations. Genome sequencing of AML has recently led to the discovery of mutations in the genes encoding isocitrate dehydrogenase (IDH1 and $I D H 2)$. IDH1 is a key cytosolic enzyme in the Krebs cycle. It catalyzes the decarboxylation of isocitrate to $\alpha$ ketoglutarate $(\alpha-\mathrm{KG})$, leading to the production of nicotinamide adenine dinucleotide phosphate (NAD-P). The isocitrate dehydrogenase 2 (IDH2) gene encodes a homologous protein which catalyzes the same reaction in mitochondria. IDH mutations have been extensively studied and are frequently found alterations in low-grade gliomas. They have also been discovered in a small subset of the highly aggressive glioblastomas, where they confer a more favorable prognosis [1-6].

IDH mutations were subsequently identified in AML and other myeloid malignancies, including MDS and MPNs $[7,8]$. All discovered $I D H$ mutations reside in the active site of the enzyme and participate in isocitrate binding [9]. They are missense alterations affecting arginine-132 (R132) in IDH1, and either the analogous arginine residue (R172), or the arginine-140 (R140) residue in the IDH2 protein [7, 1015]. The common recurrence of $I D H$ mutations in AML suggests an importance in leukemogenesis. All mutations are missense and heterozygous, suggesting that the alterations lead to a "gain of function" [9]. It has been shown that the mutant forms of IDH cannot catalyze the conversion of isocitrate to $\alpha$-KG [16]. Dang et al. reported that the mutated $\mathrm{R} 132 \mathrm{H}$ IDH1, in place of the normal process of isocitrate decarboxylation, catalyzes an NADPH-dependent reduction of $\alpha$-KG to 2-hydroxyglutarate (2-HG). 2-HG is a metabolite which is normally present at very low levels in cells [17], 2HG levels have been found to be elevated in IDH-mutant glioma samples, and Gross et al. reported that IDH1 R132 mutations also lead to production and accumulation of 2HG in AML blasts, greater than 50-fold higher than their nonmutant counterparts. Elevated 2-HG levels in IDH-WT samples led to the first discovery of IDH2 mutations, which accounted for elevated 2-HG levels in these AML cells. The elevation in 2-HG levels has also been noted in sera of patients with IDH-mutant AML [9].

Studies of $I D H$ mutations in gliomas have suggested that they are an early event in the pathogenic process [5]. Their exact mechanism in leukemogenesis of AML is uncertain. Normal IDH function appears essential for normal cell growth and proliferation. IDH1 is one of only three cytosolic proteins which contribute to NADPH production which is essential for nucleotide and lipid synthesis. Ward et al. demonstrated that siRNA silencing of the IDH1 and IDH2 proteins led to a significantly reduced proliferative capacity [15]. Some investigators have suggested that accumulation of 2-HG plays an important role in this process, the "gain of function" neomorphic enzyme activity that promotes cancer
$[9,18,19]$. Patients with a rare inherited condition called 2hydroxyglutaric aciduria have elevated levels of 2-HG with an increased propensity for brain tumors. 2-HG has indeed been shown to increase reactive oxygen species in these patients $[20,21]$. Additionally, 2-HG is homologous to $\alpha-\mathrm{KG}$ in structure and thus may bind and interfere with essential enzymes that are activated by $\alpha$-KG. Among these are prolyl hydroxylases which control the stability of and downregulate HIF- $1 \alpha$ transcription factors, implicated in the pathogenesis of multiple malignancies [9, 19, 22].

The association of IDH mutations with aberrant hypermethylation has only recently been discovered. By studying samples from 398 AML patients in an Easter Cooperative Group (ECOG) E1900 trial, we found that IDH-mutant AML is associated with consistent and aberrant hypermethylation of various promoter sites involved in myeloid differentiation and leukemogenesis [23] (Figure 1). Promoter CpG sites are extremely important in the regulation of gene expression, specifically those of genes which mediate tumor suppression and differentiation, and DNA methylation can lead to transcriptional inactivation or chromosomal instability [24, 25]. Aberrant hypermethylation has been extensive described as a pathogenic process in forms of MDS and AML [26-29]. The discovery of aberrant hypermethylation and transcriptional inactivation of loci in relation to IDHmutant AML is intriguing and significant. The potential mechanism for the hypermethylation and leukemogenesis in IDH-mutant disease may be related to downregulation of normal $\alpha$-KG levels. Multiple enzymes are dependent on $\alpha$ KG for their function, including the TET enzymes, which appear to play an important role in the differentiation of myeloid cells and promote demethylation by hydroxylating methylcytosine groups $[30,31]$.

Another recent study reported that altered $2-\mathrm{HG} / \alpha-\mathrm{KG}$ levels present in $I D H 1 / 2$ mutant cells additionally results in the inhibition of a different set of $\alpha$-KG-dependent enzymes, the Jumonji family of histone lysine demethylases (JHDM) [32]. There are 3 classes of enzymes which are known to antagonize histone methylation: (1) peptidylarginine deiminase, which removes methylarginine modifications to produce citrulline [33], (2) lysine specific demethylase 1 which removes $\mathrm{H} 3 \mathrm{~K} 4 \mathrm{me} 1 / \mathrm{H} 3 \mathrm{~K} 9 \mathrm{me} 1$ marks in a reaction requiring flavin is a cofactor [34], and the Jumonji C domain family of histone demethylases which require iron $\mathrm{Fe}(\mathrm{II})$ and $\alpha-\mathrm{KG}$ as cofactors. Unlike LSD1, which can only remove monoand dimethyl lysine modifications, the JHDMs can remove methyl groups from all three histone methylation states. So far, JHDM family members have been shown to reverse the following lysine methyl marks: H3K36me1/2 (JHDM1) [35], H3K9me1/2 (JHDM2) [36], H3K9me2/3 (JHDM3) [35], and H3K36me3 (JHDM3) [35], H3K4me2/3 (JARID1) [37], and H3K27me2/3 (UTX/JMJD3) [38]. In addition, JMJD6 has been shown to encode an arginine-specific histone demethylase which demethylates H3R2me1/2 and H4R3me1/2 [39]. All of these marks may, therefore, be affected by the presence of IDH1/2 mutations, and $\mathrm{Xu}$ et al. indeed demonstrated hypermethylation of many of these marks following introduction of $I D H 1 / 2$ mutations into cells [32] (Figure 1). These findings lend significant 


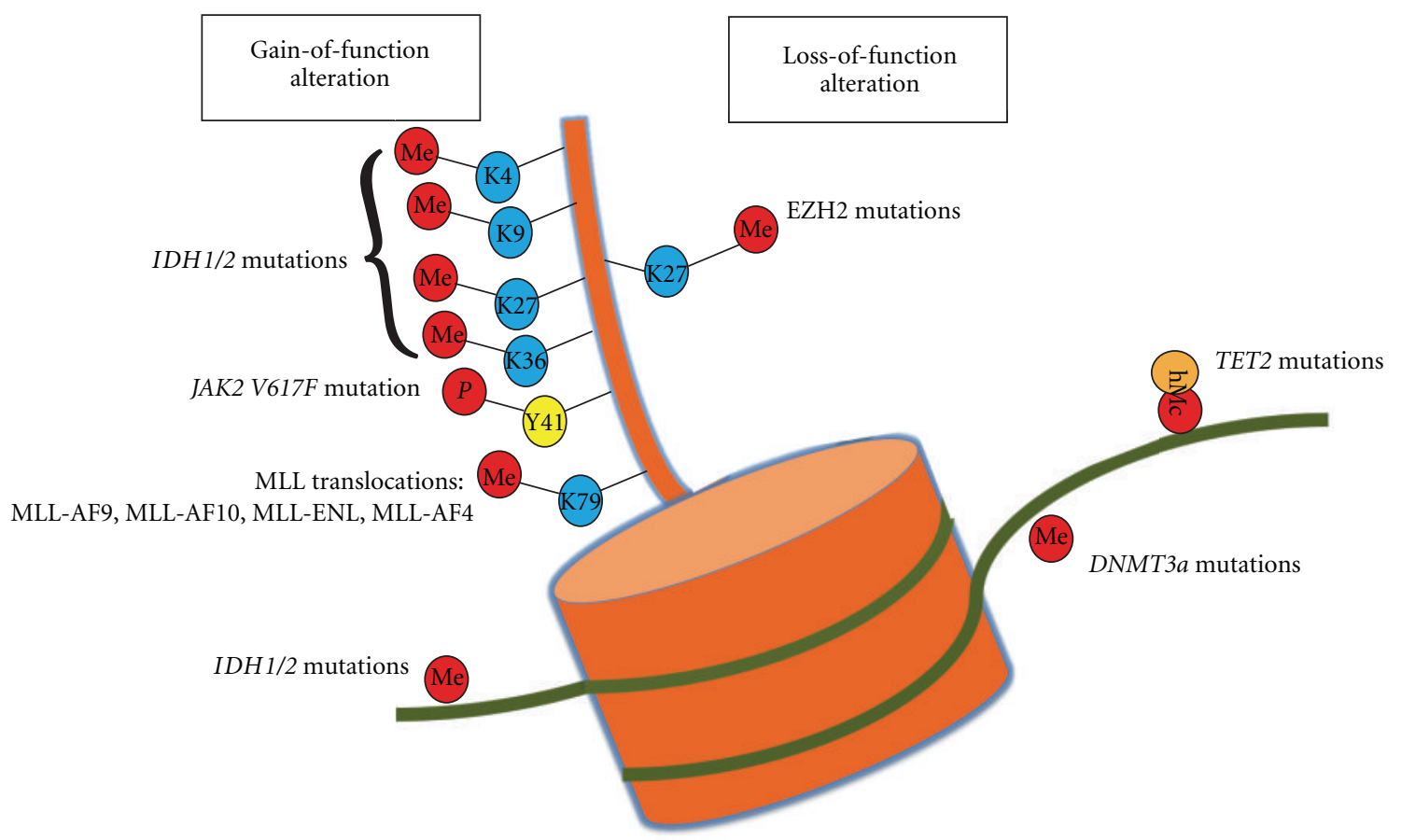

FIGURE 1: Specific histone and DNA posttranslational modifications shown to be associated with mutations in epigenetic modifiers in hematologic malignancies. Only genetic alterations which have some evidence for resulting in a gain or loss of function are displayed here. Mutations which result in the acquisition of hyperactivation or new enzymatic activity are displayed on the left of nucleosome while mutations which have evidence as resulting in a loss of enzymatic function are displayed on the right (translocations known to directly affect histone posttranslational modifications are listed in Table 1). The majority of mutations in epigenetic modifiers in myeloid malignancies recently identified are known to affect posttranslational modifications on the $\mathrm{N}$-terminal tail of histone $\mathrm{H} 3$ or at cytosines of DNA as displayed here. Currently, the function of DNMT3a mutations in AML has yet to be extensively clarified, particularly the recurrent R882 heterozygous mutations.

credence to the theory that hypermethylation of DNA and histone lysine/arginine modifications play a key role in the pathogenesis of AML in this subgroup of patients. Further work to delineate the complex epigenetic alterations to the transcriptional changes which promote leukemogenesis will be very enlightening.

Currently, our clinical use of genetics in AML for prognostication relies on (1) the use of cytogenetics to delineate patients into favorable, intermediate, or adverse cytogenetic categories and (2) molecular genotype of the genes FLT3, NPM1 (nucleophosmin 1), and CEBPA (CCAAT/enhancerbinding protein alpha) for those patients with a normal karyotype. Multiple studies have demonstrated that patients with a normal karyotype but with an internal tandem duplication of FLT3 (FLT3-ITD) have an inferior outcome compared to those without a FLT3-ITD mutation [40, 41]. In several additional studies, the presence of an NPM1 mutation with or without the presence of a FLT3-ITD mutation was associated with higher complete response and event-free survival $[42,43]$. Normal karyotype AML with mutations in CEBPA also appear to represent a subset of AML with more favorable outcome $[44,45]$. Given the identification of multiple new molecular genetic abnormalities in patients with AML, an increasing number of studies have been performed to delineate the mutational frequency and prognostic implications of IDH1/2, TET2, and DNMT3A mutations in
AML patients. The initial report of IDH1 mutations in AML found that $8.5 \%$ of AML samples contained a mutation at amino acid R132. All mutations were associated with samples that displayed intermediate risk cytogenetics, with the large majority having normal cytogenetics. With this relatively small group of 187 samples, the investigators noted no independent prognostic value on overall survival (OS), but subgroup analysis suggested adverse effects in those patients with no NPM1 mutations [7]. Ward et al. also assessed for mutations in a series of $87 \mathrm{AML}$ samples and found that $7.7 \%$ had $I D H 1$ mutations. Additionally, they found that even a larger subset of samples (15.4\%) displayed mutations in the $I D H 2$ gene. All samples with $I D H$ mutations were found to have normal karyotypes, without an effect on overall survival [15].

Larger studies, based in North America and Europe, have attempted to further investigate the prevalence and prognostic implications of these mutations. A CALGB study assessed bone marrow and peripheral blood marrow samples from 358 patients with cytogenetically normal AML. The investigators reported $I D H$ mutations in the third of their patients, with $14 \%$ having IDH1 mutations and $19 \%$ displaying $I D H 2$ mutations. AML patients with $I D H$ mutations in this study tended to be younger with lowerrisk disease as defined by a higher frequency of NPM1 mutations without the presence of FLT3-ITD mutations. 
Specifically, IDH1 mutations adversely affected disease-free survival (DFS) in this particular subgroup of patients with a favorable molecular profile. Interestingly, those patients with IDH2 mutations achieved a lower rate of complete remission with induction therapy [12].

A study from The Netherlands also found a significant prevalence of IDH mutations in 893 samples from patients with newly diagnosed AML, with $6 \%$ and $11 \%$ having IDH1 and IDH2 mutations, respectively. They also reported an association with normal karyotype AML and the NPM1 mutation. As in previous studies, no effect on OS was noted for the entire cohort of AML samples, but interestingly, in those samples without an NPM1 mutation, IDH mutations were associated with an inferior event-free survival (EFS) [10]. A subsequent study from the UK found IDH1 mutations in $8 \%$ of patient samples, using data on 1333 adult patients from the UK MRC AML10 and AML12 trials. Similar to the Dutch study, they reported a strong association with intermediate cytogenetics and the NPM1 mutation. However, in contrast to the previous study but similar to results of the CALGB trial by Marcucci et al. [12], the investigators found that an IDH1 mutation preferentially reduced survival in the cohort of patients with a coexistent NPM1 mutation [46]. These findings were supported by large studies from France and Germany, which again found that $12-16 \%$ of patients had IDH mutations, with the mutations adversely affecting rates of $\mathrm{CR}$ and survival in the subgroup of patients with CN-AML and with NPM1 mutations $[11,13]$.

However, the prognostic significance of the copresence of IDH and NPM1 mutations in CN-AML continues to be uncertain. A recent large German study reported shorter EFS and rates of CR in all AML patients with an IDH1 mutation, and specifically within the population of patients without NPM1 mutations [14]. The same authors have recently also presented their data at the annual American Society of Hematology (ASH) 2010 meeting on 526 patients with cytogenetically normal AML and found that $28.7 \%$ of patients had IDH mutations. $12.9 \%$ of patients were found to have IDH1 mutations and $15.8 \%$ had IDH2 mutations. In their survival analysis, no effect was found on OS or EFS. However, in this presentation, a trend for shorter EFS was reported in all IDH mutant patients with NPM1+/FLT3-ITD genotype, and, interestingly, a significant adverse effect on EFS in those with specifically IDH2 mutations in the NPM1+/FLT3-ITDgroup [47]. The leukemia group from M.D. Anderson in Houston, Tex, USA, also presented their data at the ASH 2010 meeting on 358 AML patients treated with induction chemotherapy. They found that IDH1 and IDH2 mutations were associated with normal karyotype and NPM1 mutations and were present in $12(7 \%)$ and $24(14 \%)$ patients and also found an IDH1G105 single-nucleotide polymorphism (SNP) alteration in 24 (14\%) patients. Overall, they found that $30 \%$ of patients had IDH alterations, but there was no association with achievement of CR, remission duration, OS or EFS, and IDH aberrations [48]. Caramazza et al. from the Mayo Clinic examined IDH mutations in 157 patients with hematologic malignancies and suggested an association with isolated trisomy 8. Eighteen IDH mutations were identified, with the majority (15) being IDH2 mutations. Seventeen of the $18 \mathrm{IDH}$ mutations occurred in myeloid malignancies, among which the large majority were MDS or AML. IDH mutations were fewer among 64 additional patients with AML or MDS without isolated trisomy 8. In MDS patients with trisomy 8 alteration, prognosis was similar between those with $I D H$ mutations and those without [49].

2.2. TET2 Mutations. TET2, the ten-eleven-translocation gene 2 , is located in chromosome 4 at band 4q24. Delhommeau and colleagues first described the presence of TET2 mutations in myeloid malignancies, by evaluating bone marrow samples from 320 patients with MDS, MPNs, and AML. TET2 defects, either mutations or deletions, were discovered in 17 of 90 patients with MDS (19\%), in 24 of 198 patients with MPN (12\%), and in 5 of 21 patients with secondary AML (24\%) [50]. Other groups have since reported a similar prevalence of TET2 mutations in myelodysplastic syndromes and other myeloid diseases [5153]. TET2 mutations were also found in the large majority (median of 96\%) of the precursor cells in the bone marrow of patients with mutations, including CD34+ progenitor cells [54]. In addition, there has also been much interest in the role of TET2 as a transformative mutation in myelodysplasia and MPNs. By analyzing the DNA of paired samples from patients with transformed, secondary AML, it was found that TET2 mutations were detected most frequently after the transformation of MPNs to AML [55].

The pathogenic mechanism of TET2 mutations in myeloid diseases has been an area of intense investigation. Strong evidence supports the integral role of TET2 in demethylation of DNA and epigenetic regulation, specifically in the conversion of 5-methylcytosine to 5-hydroxymethylcytosine $(5-\mathrm{hmC})$ [30, 31]. A recent intriguing study convincingly demonstrated that $5-\mathrm{hmC}$ is an intermediary in the process of DNA demethylation, and that TET hydroxylases are essential mediators of this process [56]. Ko et al. further demonstrated that mutations of TET2 lead to alteration and suppression of catalytic activity of the enzyme. They reported low DNA levels of 5-hmC in bone marrow samples taken from patients with TET2 mutations. In addition, the depletion of TET2 in murine models led to a suppression of differentiation of hematopoietic precursors in culture [57]. This interruption in myelopoiesis may be a necessary "hit" or alteration in the process of leukemogenesis in patients with TET-2 mutant AML.

Most recently, a variety of groups have studied the prognostic impact of TET2 mutations in MDS and AML. They appear to have a particularly high prevalence in chronic myelomonocytic leukemias, in which they have been found to be associated with significant monocytosis and poor outcomes [58]. Data on prognosis has also been presented in patients with MDS and AML, where there has been some controversy. Nibourel et al. sequenced sample DNA from 111 patients who had achieved CR after induction chemotherapy for de novo AML. They reported an overall incidence of $17 \%$ of TET 2 mutations, which were associated with NPM1 mutations but did not appear to have an impact on survival. This lack of effect on outcomes was also reported by other 
investigators [59, 60]. Another group from France reported that TET2 mutations were actually independently associated with significantly improved overall and progression-free survival in patients with MDS. Only a small percentage of patients $(7 \%)$ in this study were reported to have secondary AML [61]. In contrast, other investigators have reported a significant adverse effect on overall survival in patients with AML [51], and a recent CALGB report of 427 patients with cytogenetically normal AML found that TET2 mutations were associated with a lower rate of $\mathrm{CR}$ and shorter diseasefree and overall survival, with outcomes particularly worse for patients with favorable risk (CEPBA and NPM1 mutant) disease [62].

Perhaps, more intriguing has been the consistent finding in recent studies that TET2 mutations rarely cooccur with mutations affecting isocitrate dehydrogenase (IDH1 and IDH2) $[60,62]$. As mentioned, the possible mechanism for aberrant methylation and leukemogenesis in IDH-mutant AML may be related to the downregulation of $\alpha$-KG levels, on which TET2 enzymes depend for their activity [26]. Others have reported that the metabolite 2-HG, markedly elevated in samples of patients with IDH mutant AML, can also directly inhibit TET2 function [32]. Therefore, TET2 and $I D H$ mutations may be leukemogenic through a common mechanism, that of suppression of TET2 function. They, thus, likely act as a distinct mutational class in AML with overlapping effects on DNA methylation and leukemogenesis (Figure 1).

2.3. DNMT3A Mutations. DNA methyltransferases catalyze the methylation of cytosine residues of $\mathrm{CpG}$ dinucleotides in DNA and are encoded by the human genes DNMT1, DNMT3A, and DNMT3B. DNMT3A mutations in AML have only been recently described [63-65] and found to be present in approximately $20-22 \%$ of patients with de novo AML. Interestingly, these mutations seem to be associated with intermediate-risk AML, a finding also noted with TET2- and $I D H$-mutant disease. In contrast, no cases of favorable risk AML contained these mutations [63]. Unlike the exclusivity of TET2 and IDH mutations in recent studies of AML, DNMT3A mutations often cooccurred with IDH mutations, suggesting that these latter two mutations may not have overlapping functions in leukemogenesis.

Somatic mutations in DNMT3A have been reported as nonsense, frameshift, and missense mutations throughout the open-reading frame. However, a notable recurrent mutation in DNMT3A has been repeatedly reported in AML [63] and MDS [66] patients as a somatic missense mutation at amino acid R882. Although one study reported a decrease in DNA methylation activity of $>50 \%$ with the DNMT3A R882 mutant [64] in an in vitro methyltransferase assay, AML patient samples with DNMT3a mutations were not found to have altered total 5-methylcytosine content or altered patterns of methylation [63] (Figure 1). Equally important is the fact that the DNMT3A R882A mutation appears to occur exclusively as a heterozygous mutation suggesting a potential gain of function which may or may not require a wildtype copy of DNMT3a for altered function. Future studies examining the function of the DNMT3A R882 mutation in vitro and in vivo in the presence of wildtype DNMT3A will hopefully shed further light on a pathogenic mechanism of DNMT3a mutations in AML.

DNMT3A mutations were subsequently noted in patients with MDS and secondary AML. Walter et al., sequencing samples from 150 patients, found that 12 harbored DNMT3A mutations. Similar to the trend noted in AML, the majority of mutations were at amino acid R882. These mutations were associated with worse overall survival and rapid progression to AML, although sample size was small and transplantation status was not considered [66]. The adverse impact on survival has also been reported in patients with AML. Ley and colleagues found, in their cohort, that those with DNMT3A mutations experienced a significantly worse median overall survival of 12.3 months as compared to 41.1 months for those without mutations. Interestingly, it appeared that DNMT3A mutations accounted for the majority of the adverse effect on survival seen even in patients with FLT3-ITD alterations [63]. A more recent study from China, studying patients with acute monocytic leukemias, also reported decreased overall survival and worse outcomes in those with DNMT3A mutations [65]. Lastly, DNMT3a mutations have also recently been reported in patients with primary myelofibrosis [67]. Larger studies of DNMT3a mutations in patients with additional MPNs will be needed to further understand the clinical and/or prognostic importance of DNMT3a mutations in MPNs.

2.4. EZH2 Mutations. EZH2 is a highly conserved enzyme which serves as a histone $\mathrm{H} 3$ lysine 27 (H3K27) methyltransferase. Although EZH2 has been known to be overexpressed in several epithelial malignancies for some time, only in 2010 was it discovered that EZH2 may be mutated in hematopoietic malignancies. Curiously, a recurrent monoallelic activating mutation has been identified in EZH2 at tyrosine 641 in patients with lymphomas [68] while a series of apparent loss-of-function mutations have been found in patients with MDS and primary myelofibrosis [69-71].

The fact that EZH2 is altered by overexpression/increased activity in epithelial cancers and lymphomas, yet inactivated in myeloid malignancy, argues that the biologic consequences of alterations in $\mathrm{H} 3 \mathrm{~K} 27$ me3 may be tissue specific. At the same time, rigorous assessment of whether EZH2 mutations affect the abundance and/or the distribution of H3K27me3 in the chromatin of malignant versus paired normal nonmutated cells has not yet been published (Figure 1). In addition, investigation of the effects of EZH2 mutations on alterations in DNA methylation may be particularly important given that EZH2 physically interacts with DNA methyltransferases 1-3 [72], and data suggests that H3K27 methylation is a necessary prerequisite for DNA promoter methylation [72].

From a clinical standpoint, inactivation of EZH2 by loss or mutation in MDS is enlightening as cytogenetic abnormalities of chromosome 7 which have been long recognized in MDS and AML and linked to adverse outcome $[73,74]$. In fact, several studies suggest that MDS patients with EZH2 mutations have worsened overall survival compared to those without EZH2 mutations, regardless of gross 
cytogenetic findings [70, 71]. Larger studies incorporating EZH2 mutations in light of additional genetic abnormalities will be needed to further clarify the prognostic importance of EZH2 mutations in MDS and MPNs and are ongoing.

\section{The Prospect of Novel Therapeutic Agents Targeting Epigenetic Modifiers in Myeloid Malignancies}

3.1. DNA Methyltransferase Inhibitors (DNMTIs). The first three epigenetic targeted therapeutics which have been FDA approved for use in the United States include 2 drugs targeting DNMTs (azacitidine (AZA) and decitabine (5aza- $2^{\prime}$-deoxycytidine)) as well as one histone deacetylase inhibitor (vorinostat). Although the use of DNMTIs has proven useful in the therapy of high-risk MDS as well as in AML, there are several questions which have lingered regarding the use of these therapies: (1) what is the ideal dose and schedule of DNMTIs? (2) what is the true mechanism of action of the nucleoside DNMTIs? (3) are there biomarkers which can be used for predicting response and/or resistance to DNMTIs? and (4) can we develop nonnucleoside direct inhibitors of DNMTs?

Despite questions regarding use and schedule of DNMTIs, there have been several interesting new developments in this class of therapeutics. Within the original category of nucleoside analog DNMTIs, an oral formulation of AZA has recently been developed [75]. Parenteral azacitidine is approved for administration at $75 \mathrm{mg} / \mathrm{m}^{2}$ for 7 days every 28 days, and this dose is believed to result in DNA hypomethylation as well as cytotoxicity. Using several assays for DNA methylation, this dose of 5-aza has been shown to result in maximal DNA hypomethylation at approximately day 15 with gradual return of DNA methylation back to baseline around the time of next cycle [76]. Given this, use of orally administered AZA on a more frequent schedule may result in altered effects on DNA methylation and cellular cytotoxicity and holds the potential for greater therapeutic efficacy. The initial phase I trial of oral AZA on a 7 days schedule revealed that the drug is bioavailable, safe, and clinically active in patients with MDS and AML. At the 2010 ASH meeting, results of the multicenter phase I study of extended oral AZA schedules revealed that oral azacitidine on a 14- or 21-day schedule is well tolerated, with no AZA accumulation, and promising clinical responses were observed [75].

The direct cytotoxic effects of the clinically utilizing DNMTIs as well as their chemical instability have prompted continuous rationale for developing additional DNMTIs. A third nucleoside DNMTI which has been under development for some time is the cytidine analogue zebularine. Although zebularine has a similar mechanism of action as decitabine and azacitidine, resulting in the depletion of DNMTs through covalent bonding with DNMTs, zebularine has a much longer half-life making oral administration of the drug possible [77, 78]. Additionally, zebularine appears to be selectively incorporated into malignant and not normal cells admixed with tumor in at least one setting, a property not seen with decitabine or AZA [79]. Despite these properties, one limitation to the development of zebularine for clinical use has been the fact that higher concentrations of zebularine are needed to obtain similar levels of demethylation in cells in comparison with azacitidine and decitabine [78]. Further preclinical works addressing the practicality of the drug as a clinical therapeutic agent are ongoing.

In addition to the nucleoside DNMTIs, there has been considerable efforts at developing nonnucleoside targeted molecules to directly inhibit individual DNMTs. One approach has been the development of antisense oligonucleotides targeting DNMT1 for in vivo use. One such molecule, the phosphorothioate antisense oligonucleotide MG98, was developed based on its ability to knockdown DNMT1 expression in various model systems [80, 81]. However, phase I clinical trials of MG98 in solid and hematopoietic tumors was disappointing with very little consistent knock down of DNMT1 mRNA in patients [82, 83]. This likely resulted from inefficient intracellular uptake of MG98.

Rational design of small molecules targeting DNMTs through noncovalent interactions with the catalytic sites of these enzymes has resulted in the development and characterization of several test compounds. The first rationally designed DNMT1 inhibitor is RG108 which was designed utilizing a three-dimensional model of the human DNMT1 catalytic pocket [84]. RG108 has comparable demethylating activity to zebularine but appears to be less active than azacitidine and decitabine. Several additional small-molecular inhibitors of DNMTs have more recently been found within the NCI open database of compounds through a similar screening approach which led to the discovery of RG108 [85]. Further preclinical characterization of all of these compounds is underway.

The use of DNMTIs in patients with high-risk MDS and AML has proven that while durable complete remissions are possible with these drugs, responses can be quite variable with no current routinely used clinical parameter known to predict likelihood of response to therapy. With the discovery of mutations in TET2 in these patients and the postulated pathogenic mechanism of TET2 mutations in MDS/AML, it has been hypothesized that TET2-mutated patients may have higher rates of response to DNMTIs. This has recently been suggested by a small French study of 86 patients with MDS and secondary AML. The investigators reported that those with TET2 mutations experienced a response rate (RR) of $82 \%$ to AZA in comparison to the TET2-wt group, which had a significantly lower RR of $45 \%$. However, there was no effect on survival parameters, and the study group was quite heterogeneous with few additional genetic parameters studied [86]. In contrast, other groups have found that TET2 alterations in a similar cohort of patients may actually predict for decreased responsiveness to demethylating therapies [87]. The small number of patients included in these studies and the limited genetic characterization of the patients must be considered. Larger studies with more comprehensive genetic evaluation will be critical in determining if mutations in genetic factors suspected to be important in regulating DNA methylation 
(TET2, IDH1/2, and DNMT3a mutations, amongst others) affect response to DNMTIs.

\section{The Prospect for Novel Histone Methyltransferase Inhibitors in Myeloid Leukemias: DOT1L Inhibition and Rational Design of Protein Methyltransferase Inhibitors}

Recent discovery of the potential importance of aberrant hypermethylation of histone lysines in the pathogenesis of myeloid leukemias driven by MLL-translocations [88], NUP98-NSD1 translocations [89], and possibly IDH1/2mutant disorders [32] suggests the possibly of targeting histone lysine methyltransferases in myeloid leukemias.

One novel and exciting prospect utilizing this therapeutic rationale is the study of DOT1L-targeted therapy for the selective treatment of MLL-translocated leukemias. In addition to the recently identified mutations in epigenetic modifiers in MDS and AML, a number of translocations disrupting the normal activity of epigenetic modifiers in myeloid malignancies have been recognized for a longer period of time (Table 1). Key amongst the frequent translocations altering the activity of an epigenetic modifiers in AML includes translocations involving mixed lineage leukemia 1 (MLL1) which occur in at least $10 \%$ of adult AML patients and $>70 \%$ of infant acute leukemias. MLL1 normally serves as an histone $\mathrm{H} 3$ lysine 4 (H3K4) methyltransferase. MLL1 translocations result in fusion of the N terminus of MLL1 to one of $>60$ different translocation partners [90]. In a recent landmark survey of MLL rearrangements, 760 MLLrearranged biopsy samples were reviewed and 104 different MLL rearrangements were found in adult and pediatric acute leukemia patients [90]. However, amongst AML patients with MLL rearrangements, $77 \%$ were accounted for by one of 7 translocations: MLL-AF9 (30.4\%), MLL-AF10 (14.5\%), MLL-ELL (10.9\%), MLL-AF6 (10.1\%), MLL-ENL (5.4\%), MLL-AF17 (2.9\%), and MLL-SEPT6 (2.5\%). Many of these same MLL rearrangements are also common in acute lymphoblastic leukemia (ALL) along with MLL-AF4 which is the most common MLL rearrangement in ALL (accounting for $66 \%$ of MLL-rearranged ALL cases) [90].

Importantly, the four most frequent MLL translocations (MLL-AF4, MLL-AF9, MLL-AF10, and MLL-ENL) result in recruitment of DOT1L (disruptor of telomeric silencing 1like) to the fusion protein and acquisition of histone 3 lysine 79 (H3K79) methyltransferase activity (Figure 1). A number of studies using both shRNA for DOT1L and conditional deletion of DOT1L have recently shown that the H3K79 methyltransferase activity is critical for leukemogenesis induced by MLL-fusion proteins $[91,92]$. This has led to the concept of developing targeted therapy for DOT1L inhibition in the therapy of MLL-translocated leukemias. At the 2010 ASH meeting, investigators at Epizyme Inc. presented the initial results from in vitro studies of the first DOT1L inhibitor, EPZ01 [93]. EPZ01 acts as a competitive inhibitor of the cofactor S-adenosylmethionine (SAM), the universal methyl donor for all enzymatic methyltransferase reactions.
Despite the ubiquity of SAM in protein methyltransferase reactions, EPZ01 is reported to have a 500-fold selectivity for DOT1L over other lysine histone and arginine methyltransferases. Consistent with this, the investigators revealed selective killing of EPZ01 for leukemia cell lines bearing MLL1 translocations over non-MLL rearranged cell lines. DOT1L-inhibition appeared to downregulate H3K79me3 abundance globally and at critical loci, serving both as proof of concept of the mechanism of activity and potentially as a biomarker of response [93].

As mentioned earlier, MLL rearrangements are also frequent in ALL with MLL-AF4 translocation being the most frequent MLL rearrangement in ALL [90]. Although two initial transgenic mouse models of MLL-AF4 fusion gene overexpression did not result in the development of acute leukemia, a conditional knockin model of MLL-AF4 [94] as well as a retroviral transplantation model of MLLAF4 [95] did result in the development of ALL. Moreover, mice with overexpression of MLL-AF4 in the conditional knockin model by Krivstov et al. were clearly distinguished by increases in H3K79me3 indicating a clear link between the presence of MLL-AF4 fusion oncoprotein and acquisition of increased H3K79 methyltransferase activity. In addition, recent purification of the MLL-AF4 complex has clearly indicated binding of DOT1L to this complex [96]. Equally important, use of an shRNA against DOT1L inhibited the expression of several genes critical for MLL-AF4-mediated oncogenesis in the MLL-AF4-conditional knockin model underscoring the potential importance of DOT1L inhibition in the therapy of ALL with MLL-AF4 rearrangement [94].

One key question which must be further addressed in the preclinical development of DOT1L-targeted therapy is the question of potential adverse ramifications of DOT1L inhibition. Jo et al. recently reported that mice bearing conditional disruption of DOT1L-developed pancytopenia and failure of hematopoietic homeostasis revealing a critical role of DOT1L in normal hematopoiesis [91]. At the same meeting, Bernt et al. also developed a conditional deletion model of DOT1L in vivo using Vav-Cre technology for DOT1L deletion in the adult hematopoietic and endothelial cells but also in the germline [92]. DOT1L $\mathrm{L}^{-/-}$mice in this system were born at Mendelian rations and with blood counts at lower border of normal range. In addition, cardiacspecific deletion of DOT1L resulted in increased mortality in mice due to cardiac dysfunction which closely resembled human dilated cardiomyopathy [97]. Interestingly, Nguyen et al. further discovered that DOT1L is downregulated in patients with idiopathic dilated cardiomyopathy, and the cardiac phenotype in mice could be rescued by expression of dystrophin [97]. Further characterization of these mouse models and use of DOT1L inhibitors in preclinical in vivo testing will hopefully clarify the potential utility and safety of this very promising new therapeutic strategy.

Development of other specific inhibitors of histone methyltransferases holds promise in myeloid leukemias. The challenges for this prospect have been twofold: (1) knowledge of the genome-wide and locus-specific effects of histone modifications due to direct genetic abnormalities found in myeloid leukemia patients has been less clear for the 
TABLE 1: Translocations directly affecting histone modifying enzymes or recruitment of histone modifying enzymes in patients with myeloid malignancies.

\begin{tabular}{|c|c|}
\hline Gene & Effects of translocation on histone posttranslational modifications \\
\hline MLL1 & $\begin{array}{l}\text { MLL1 normally serves as an H3K4 methyltransferase. MLL-AF4, MLL-AF9, MLL-AF10, and MLL-ENL } \\
\text { translocations result in loss of the SET domain and recruitment of DOT1L binding resulting in acquisition of } \\
\text { H3K79 methyltransferase activity. }\end{array}$ \\
\hline CBP & $\begin{array}{l}\text { The histone acetyltransferase CBP has been reported to undergo translocation with MOZ in AML. This results in } \\
\text { the disruption of CBP's normal acetyltransferase activity and also in recruitment of CBP to MOZ-regulated gene } \\
\text { promoters. MOZ also contains a putative acetyltransferase domain which may be affected in this translocation. } \\
\text { CBP is also an occasional translocation partner with MLL1. }\end{array}$ \\
\hline NSD1 & $\begin{array}{l}\text { The H3K36 methyltransferase NSD1 has been rarely reported to undergo translocation with NUP98 in AML. This } \\
\text { translocation does not abrogate H3K36 methyltransferase activity of NSD1 but rather promotes aberrant H3K36 } \\
\text { methylation at specific loci which promotes leukemogenesis. }\end{array}$ \\
\hline P300 & $\begin{array}{l}\text { The histone acetyltransferase p300 is an occasional translocation partner with MLL1 in AML. This translocation } \\
\text { preserves the majority of the coding sequence of p300, and the direct transcriptional and histone effects of this } \\
\text { translocation are not well characterized. Interesting p } 300 \text { - and CBP-MLL translocations appear to be significantly } \\
\text { associated with therapy-related AML rather than de novo AML suggesting a potential difference in the } \\
\text { pathogenesis of these } 2 \text { subtypes of AML. }\end{array}$ \\
\hline AML1 & $\begin{array}{l}\text { Translocations involving AML1 are characteristic of a proportion of patients with core-binding factor leukemias. } \\
\text { Normally the C terminus of AML1 interacts with the histone acetyltransferase p300 and recruits p300 to specific } \\
\text { loci bound by the N terminus of AML1. However, in the common translocation } \mathrm{t}(8 ; 21)(\mathrm{q} 22 ; \mathrm{q} 22) \text {, the } \mathrm{C} \text { terminus } \\
\text { of AML1 is lost and replaced with the C terminus of ETO which attracts a corepressor complex with histone } \\
\text { deacetylase activity (N-CoR/Sin3/HDAC complex). }\end{array}$ \\
\hline $\operatorname{RAR} \alpha$ & $\begin{array}{l}\text { The characteristic translocation of acute promyelocytic leukemia, t(15;17)(q21;q21) fuses PML with RAR } \alpha \text {. } \\
\text { Recently it has been demonstrated that one of the critical aspects of PML-RAR } \alpha \text {-induced oncogenesis is aberrant } \\
\text { downregulation of histone H3 acetylation by the PML-RAR } \alpha \text { fusion protein. Normally, in the presence of its } \\
\text { ligand retinoic acid, RAR } \alpha \text { functions as a transcriptional activator. However, when ligand is not present, RARa } \\
\text { functions as a transcriptional repressor through recruitment of HDACs. The PML-RAR } \alpha \text { fusion protein results in } \\
\text { constitutive HDAC activity and aberrant target gene repression. Pharmacologic doses of ATRA appear to greatly } \\
\text { increase histone H3 acetylation, and this, in part, serves to reverse some of the oncogenic effects of the PML-RAR } \alpha \\
\text { fusion protein. }\end{array}$ \\
\hline
\end{tabular}

majority of non-MLL translocated patients. For instance, loss-of-function mutations in the H3K27 methyltransferase EZH2 has been recently found in patients with MDS and primary myelofibrosis [69-71]. At the same time, loss of the H3K27 demethylase UTX has also been suggested to occur in some of the same disorders making the rationale for targeted changes affecting $\mathrm{H} 3 \mathrm{~K} 27$ methylation hard to understand [98]. (2) In addition, from a drug development standpoint, potent and selective inhibitors of histone protein methyltransferases have only recently begun. One strategy mentioned earlier is the development of small-molecule inhibitors of the SAM-binding pocket, a universal feature of protein methyltransferase somewhat analogous to the targeting of the ATP-binding pockets of protein kinases. In fact, the first selective and potent protein methyltransferase inhibitor was recently reported using this strategy [99]. This molecule serves to inhibit the arginine methyltransferase CARM1, a protein overexpressed and thought to be important in the pathogenesis of prostate and breast carcinomas $[100,101]$. Although empiric use of these medications in early-phase clinical trials has been utilized previously with some success, in-depth characterization of histone posttranslational modifications in patients with MDS/AML may shed light on rational strategy for specific histone methyltransferase inhibition as a therapeutic strategy in these disorders.

\section{Conclusion}

The exciting discovery of new genetic abnormalities in patients with myeloid malignancies holds the promise for furthering our understanding of the pathogenesis of these disorders but also in refining our risk stratification and therapeutic management of patients. As highlighted here, a series of studies have rapidly suggested that mutations in TET2, IDH1/2, and DNMT3a will likely refine our current prognostication of patients with AML if borne out repeatedly in large prospective trials of AML patients. Moreover, given the suggested effects of these genetic abnormalities on DNA methylation, the potential importance of these mutations on affecting response to DNMTIs will need to be more thoroughly investigated. The effects of the recurrent DNMT3a R882 mutation will particularly need to be scrutinized given its frequency and the fact that it is always present as a heterozygous mutation. Furthering our understanding of the specific altered epigenetic marks placed by genetic abnormalities in MDS/AML patients is critical as it may result in the development of novel epigenetic 
targeted therapeutics. The clearest example of this currently is the exciting development of DOT1L inhibitors for MLLtranslocated leukemias described here. Further characterization of DOT1L deficiency/inhibition in a variety of in vivo models is greatly anticipated. Moreover, development of additional protein methyltransferase inhibitors is likely forthcoming and prompts for greater understanding of the epigenetic alterations present in patients with MDS and AML.

\section{References}

[1] F. Ducray, Y. Marie, and M. Sanson, "IDH1 and IDH2 mutations in gliomas," The The New England Journal of Medicine, vol. 360, p. 2248, 2009.

[2] C. Hartmann, J. Meyer, J. Balss et al., "Type and frequency of IDH1 and IDH2 mutations are related to astrocytic and oligodendroglial differentiation and age: a study of 1,010 diffuse gliomas," Acta Neuropathologica, vol. 118, no. 4, pp. 469-474, 2009.

[3] D. W. Parsons, S. Jones, X. Zhang et al., "An integrated genomic analysis of human glioblastoma multiforme," Science, vol. 321, no. 5897, pp. 1807-1812, 2008.

[4] M. Sanson, Y. Marie, S. Paris et al., "Isocitrate dehydrogenase 1 codon 132 mutation is an important prognostic biomarker in gliomas," Journal of Clinical Oncology, vol. 27, no. 25, pp. 4150-4154, 2009.

[5] T. Watanabe, S. Nobusawa, P. Kleihues, and H. Ohgaki, "IDH1 mutations are early events in the development of astrocytomas and oligodendrogliomas," American Journal of Pathology, vol. 174, no. 4, pp. 1149-1153, 2009.

[6] Y. Sonoda, T. Kumabe, T. Nakamura et al., "Analysis of IDH1 and IDH2 mutations in Japanese glioma patients," Cancer Science, vol. 100, no. 10, pp. 1996-1998, 2009.

[7] E. R. Mardis, L. Ding, D. J. Dooling et al., "Recurring mutations found by sequencing an acute myeloid leukemia genome," The New England Journal of Medicine, vol. 361, no. 11, pp. 1058-1066, 2009.

[8] A. Tefferi, T. L. Lasho, O. Abdel-Wahab et al., "IDH1 and IDH2 mutation studies in 1473 patients with chronic-, fibrotic- or blast-phase essential thrombocythemia, polycythemia vera or myelofibrosis," Leukemia, vol. 24, no. 7, pp. 1302-1309, 2010.

[9] S. Gross, R. A. Cairns, M. D. Minden et al., "Cancerassociated metabolite 2-hydroxyglutarate accumulates in acute myelogenous leukemia with isocitrate dehydrogenase 1 and 2 mutations," Journal of Experimental Medicine, vol. 207, no. 2, pp. 339-344, 2010.

[10] S. Abbas, S. Lugthart, F. G. Kavelaars et al., "Acquired mutations in the genes encoding IDH1 and IDH2 both are recurrent aberrations in acute myeloid leukemia: prevalence and prognostic value," Blood, vol. 116, no. 12, pp. 2122-2126, 2010.

[11] N. Boissel, O. Nibourel, A. Renneville et al., "Prognostic impact of isocitrate dehydrogenase enzyme isoforms 1 and 2 mutations in acute myeloid leukemia: a study by the acute leukemia French association group," Journal of Clinical Oncology, vol. 28, no. 23, pp. 3717-3723, 2010.

[12] G. Marcucci, K. Maharry, Y. Z. Wu et al., "IDH1 and IDH2 gene mutations identify novel molecular subsets within de novo cytogenetically normal acute myeloid leukemia: a cancer and leukemia group B study," Journal of Clinical Oncology, vol. 28, no. 14, pp. 2348-2355, 2010.
[13] P. Paschka, R. F. Schlenk, V. I. Gaidzik et al., "IDH1 and IDH2 mutations are frequent genetic alterations in acute myeloid leukemia and confer adverse prognosis in cytogenetically normal acute myeloid leukemia with NPM1 mutation without FLT3 internal tandem duplication," Journal of Clinical Oncology, vol. 28, no. 22, pp. 3636-3643, 2010.

[14] S. Schnittger, C. Haferlach, M. Ulke, T. Alpermann, W. Kern, and T. Haferlach, "IDH1 mutations are detected in $6.6 \%$ of 1414 AML patients and are associated with intermediate risk karyotype and unfavorable prognosis in adults younger than 60 years and unmutated NPM1 status," Blood, vol. 116, no. 25, pp. 5486-5496, 2010.

[15] P. S. Ward, J. Patel, D. R. Wise et al., "The common feature of leukemia-associated IDH1 and IDH2 mutations is a neomorphic enzyme activity converting $\alpha$-ketoglutarate to 2hydroxyglutarate," Cancer Cell, vol. 17, no. 3, pp. 225-234, 2010.

[16] H. Yan, D. W. Parsons, G. Jin et al., "IDH1 and IDH2 mutations in gliomas," The New England Journal of Medicine, vol. 360, no. 8, pp. 765-773, 2009.

[17] L. Dang, D. W. White, S. Gross et al., "Cancer-associated IDH1 mutations produce 2-hydroxyglutarate," Nature, vol. 462, no. 7274, pp. 739-744, 2009.

[18] Z. J. Reitman, D. W. Parsons, and H. Yan, "IDH1 and IDH2: not your typical oncogenes," Cancer Cell, vol. 17, no. 3, pp. 215-216, 2010.

[19] Z. J. Reitman and H. Yan, "Isocitrate dehydrogenase 1 and 2 mutations in cancer: alterations at a crossroads of cellular metabolism," Journal of the National Cancer Institute, vol. 102, no. 13, pp. 932-941, 2010.

[20] S. Kölker, V. Pawlak, B. Ahlemeyer et al., "NMDA receptor activation and respiratory chain complex $\mathrm{V}$ inhibition contribute to neurodegeneration in D-2-hydroxyglutaric aciduria," European Journal of Neuroscience, vol. 16, no. 1, pp. 21-28, 2002.

[21] A. Latini, K. Scussiato, R. B. Rosa et al., "D-2-hydroxyglutaric acid induces oxidative stress in cerebral cortex of young rats," European Journal of Neuroscience, vol. 17, no. 10, pp. 20172022, 2003.

[22] C. Frezza, D. A. Tennant, and E. Gottlieb, "IDH1 mutations in gliomas: when an enzyme loses its grip," Cancer Cell, vol. 17, no. 1, pp. 7-9, 2010.

[23] M. E. Figueroa, O. Abdel-Wahab, C. Lu et al., "Leukemic IDH1 and IDH2 mutations result in a hypermethylation phenotype, disrupt TET2 function, and impair hematopoietic differentiation," Cancer Cell, vol. 18, no. 6, pp. 553-567, 2010.

[24] J. Boultwood and J. S. Wainscoat, "Gene silencing by DNA methylation in haematological malignancies," British Journal of Haematology, vol. 138, no. 1, pp. 3-11, 2007.

[25] G. Leone, L. Teofili, M. T. Voso, and M. Lübbert, "DNA methylation and demethylating drugs in myelodysplastic syndromes and secondary leukemias," Haematologica, vol. 87, no. 12, pp. 1324-1341, 2002.

[26] M. E. Figueroa, S. Lugthart, Y. Li et al., "DNA methylation signatures identify biologically distinct subtypes in acute myeloid leukemia," Cancer Cell, vol. 17, no. 1, pp. 13-27, 2010.

[27] S. D. Gore, S. Baylin, E. Sugar et al., "Combined DNA methyltransferase and histone deacetylase inhibition in the treatment of myeloid neoplasms," Cancer Research, vol. 66, no. 12, pp. 6361-6369, 2006.

[28] R. Khan, J. Schmidt-Mende, M. Karimi et al., "Hypomethylation and apoptosis in 5-azacytidine-treated myeloid cells," Experimental Hematology, vol. 36, no. 2, pp. 149-157, 2008. 
[29] S. Lugthart, M. E. Figueroa, E. Bindels et al., "Aberrant DNA hypermethylation signature in acute myeloid leukemia directed by EVI1," Blood, vol. 117, no. 1, pp. 234-241, 2011.

[30] S. Ito, A. C. D’Alessio, O. V. Taranova, K. Hong, L. C. Sowers, and Y. Zhang, "Role of Tet proteins in $5 \mathrm{mC}$ to $5 \mathrm{hmC}$ conversion, ES-cell self-renewal and inner cell mass specification," Nature, vol. 466, no. 7310, pp. 1129-1133, 2010.

[31] M. Tahiliani, K. P. Koh, Y. Shen et al., "Conversion of 5-methylcytosine to 5-hydroxymethylcytosine in mammalian DNA by MLL partner TET1," Science, vol. 324, no. 5929, pp. 930 $935,2009$.

[32] W. Xu, H. Yang, Y. Liu et al., "Oncometabolite 2-hydroxyglutarate is a competitive inhibitor of $\alpha$-ketoglutaratedependent dioxygenases," Cancer Cell, vol. 19, no. 1, pp. 1730, 2011.

[33] Y. Wang, J. Wysocka, J. Sayegh et al., "Human PAD4 regulates histone arginine methylation levels via demethylimination," Science, vol. 306, no. 5694, pp. 279-283, 2004.

[34] Y. Shi, F. Lan, C. Matson et al., "Histone demethylation mediated by the nuclear amine oxidase homolog LSD1," Cell, vol. 119, no. 7, pp. 941-953, 2004.

[35] R. J. Klose, K. Yamane, Y. Bae et al., "The transcriptional repressor JHDM3A demethylates trimethyl histone $\mathrm{H} 3$ lysine 9 and lysine 36," Nature, vol. 442, no. 7100, pp. 312-316, 2006.

[36] K. Yamane, C. Toumazou, Y. I. Tsukada et al., "JHDM2A, a JmjC-containing H3K9 demethylase, facilitates transcription activation by androgen receptor," Cell, vol. 125, no. 3, pp. 483-495, 2006.

[37] D. J. Seward, G. Cubberley, S. Kim et al., "Demethylation of trimethylated histone H3 Lys4 in vivo by JARID1 JmjC proteins," Nature Structural and Molecular Biology, vol. 14, no. 3, pp. 240-242, 2007.

[38] K. Agger, P. A. C. Cloos, J. Christensen et al., "UTX and JMJD3 are histone H3K27 demethylases involved in HOX gene regulation and development," Nature, vol. 449, no. 7163, pp. 731-734, 2007.

[39] B. Chang, Y. Chen, Y. Zhao, and R. K. Bruick, "JMJD6 is a histone arginine demethylase," Science, vol. 318, no. 5849, pp. 444-447, 2007.

[40] R. F. Schlenk, K. Döhner, J. Krauter et al., "Mutations and treatment outcome in cytogenetically normal acute myeloid leukemia," The New England Journal of Medicine, vol. 358, no. 18, pp. 1909-1918, 2008.

[41] S. Fröhling, R. F. Schlenk, J. Breitruck et al., "Prognostic significance of activating FLT3 mutations in younger adults (16 to 60 years) with acute myeloid leukemia and normal cytogenetics: a study of the AML study group Ulm," Blood, vol. 100, no. 13, pp. 4372-4380, 2002.

[42] S. Schnittger, C. Schoch, W. Kern et al., "Nucleophosmin gene mutations are predictors of favorable prognosis in acute myelogenous leukemia with a normal karyotype," Blood, vol. 106, no. 12, pp. 3733-3739, 2005.

[43] K. Döhner, R. F. Schlenk, M. Habdank et al., "Mutant nucleophosmin (NPM1) predicts favorable prognosis in younger adults with acute myeloid leukemia and normal cytogenetics: interaction with other gene mutations," Blood, vol. 106, no. 12, pp. 3740-3746, 2005.

[44] S. Fröhling, R. F. Schlenk, I. Stolze et al., "CEBPA mutations in younger adults with acute myeloid leukemia and normal cytogenetics: prognostic relevance and analysis of cooperating mutations," Journal of Clinical Oncology, vol. 22, no. 4, pp. 624-633, 2004.
[45] C. Preudhomme, C. Sagot, N. Boissel et al., "Favorable prognostic significance of CEBPA mutations in patients with de novo acute myeloid leukemia: a study from the Acute Leukemia French Association (ALFA)," Blood, vol. 100, no. 8, pp. 2717-2723, 2002.

[46] C. L. Green, C. M. Evans, R. K. Hills, A. K. Burnett, D. C. Linch, and R. E. Gale, "The prognostic significance of IDH1 mutations in younger adult patients with acute myeloid leukemia is dependent on FLT3/ITD status," Blood, vol. 116, no. 15, pp. 2779-2782, 2010.

[47] S. Schnittger, C. Haferlach, T. Alpermann, W. Kern, and T. Haferlach, "IDH mutations can be detected In $28.7 \%$ of all normal karyotype AML and have unfavourable impact on the NPM1+/FLT3-ITD- genotype," Blood, vol. 116, 2010, ASH Annual Meeting Abstracts, Abstract 102.

[48] F. Ravandi, K. P. Patel, R. Luthra et al., "Prognostic significance of mutations in isocitrate dehydrogenase (IDH) enzyme isoforms 1 and 2 and single nucleotide polymorphisms (SNP) in IDH1, in patients with acute myeloid leukemia treated with high dose cytarabine and idarubicin induction," Blood, vol. 116, 2010, ASH Annual Meeting Abstracts, Abstract 2706.

[49] D. Caramazza, T. Lasho, C. Finke et al., "IDH mutations and trisomy 8 in myelodysplastic syndromes and acute myeloid leukemia," Blood, vol. 116, 2010, ASH Annual Meeting Abstracts, Abstract 4009.

[50] F. Delhommeau, S. Dupont, V. D. Valle et al., "Mutation in TET2 in myeloid cancers," The New England Journal of Medicine, vol. 360, no. 22, pp. 2289-2301, 2009.

[51] O. Abdel-Wahab, A. Mullally, C. Hedvat et al., "Genetic characterization of TET1, TET2, and TET3 alterations in myeloid malignancies," Blood, vol. 114, no. 1, pp. 144-147, 2009.

[52] A. M. Jankowska, H. Szpurka, R. V. Tiu et al., "Loss of heterozygosity $4 \mathrm{q} 24$ and TET2 mutations associated with myelodysplastic/myeloproliferative neoplasms," Blood, vol. 113, no. 25, pp. 6403-6410, 2009.

[53] A. Tefferi, A. Pardanani, K. H. Lim et al., "TET2 mutations and their clinical correlates in polycythemia vera, essential thrombocythemia and myelofibrosis," Leukemia, vol. 23, no. 5, pp. 905-911, 2009.

[54] S. M. C. Langemeijer, R. P. Kuiper, M. Berends et al., "Acquired mutations in TET2 are common in myelodysplastic syndromes," Nature Genetics, vol. 41, no. 7, pp. 838-842, 2009.

[55] O. Abdel-Wahab, T. Manshouri, J. Patel et al., "Genetic analysis of transforming events that convert chronic myeloproliferative neoplasms to leukemias," Cancer Research, vol. 70, no. 2, pp. 447-452, 2010.

[56] J. U. Guo, Y. Su, C. Zhong, G. L. Ming, and H. Song, "Hydroxylation of 5-methylcytosine by TET1 promotes active DNA demethylation in the adult brain," Cell, vol. 145, no. 3, pp. 423-434, 2011.

[57] M. Ko, Y. Huang, A. M. Jankowska et al., "Impaired hydroxylation of 5-methylcytosine in myeloid cancers with mutant TET2," Nature, vol. 468, no. 7325, pp. 839-843, 2010.

[58] O. Kosmider, V. Gelsi-Boyer, M. Ciudad et al., "TET2 gene mutation is a frequent and adverse event in chronic myelomonocytic leukemia," Haematologica, vol. 94, no. 12, pp. 1676-1681, 2009.

[59] O. Nibourel, O. Kosminder, M. Cheok et al., "Association of TET2 alterations with NPM1 mutations and prognostic value in de novo Acute Myeloid Leukemia (AML)," Blood, vol. 114, no. 22, 2009. 
[60] V. Gaidzik, R. F. Schlenk, P. Paschka et al., "TET2 mutations in Acute Myeloid Leukemia (AML): results on 783 patients treated within the AML HD98A study of the AML Study Group (AMLSG)," Blood, vol. 116, 2010.

[61] O. Kosmider, V. Gelsi-Boyer, M. Cheok et al., "TET2 mutation is an independent favorable prognostic factor in myelodysplastic syndromes (MDSs)," Blood, vol. 114, no. 15, pp. 3285-3291, 2009.

[62] K. H. Metzeler, K. Maharry, M. D. Radmacher et al., "TET2 mutations improve the new European LeukemiaNet risk classification of acute myeloid leukemia: a cancer and leukemia group B study," Journal of Clinical Oncology, vol. 29, no. 10, pp. 1373-1381, 2011.

[63] T. J. Ley, L. Ding, M. J. Walter et al., "DNMT3A mutations in acute myeloid leukemia," The New England Journal of Medicine, vol. 363, no. 25, pp. 2424-2433, 2010.

[64] Y. Yamashita, J. Yuan, I. Suetake et al., "Array-based genomic resequencing of human leukemia," Oncogene, vol. 29, no. 25, pp. 3723-3731, 2010.

[65] X. J. Yan, J. Xu, Z. H. Gu et al., "Exome sequencing identifies somatic mutations of DNA methyltransferase gene DNMT3A in acute monocytic leukemia," Nature Genetics, vol. 43, no. 4, pp. 309-315, 2011.

[66] M. J. Walter, L. Ding, D. Shen et al., "Recurrent DNMT3A mutations in patients with myelodysplastic syndromes," Leukemia. In press.

[67] O. Abdel-Wahab, A. Pardanani, R. Rampal, T. L. Lasho, R. L. Levine, and A. Tefferi, "DNMT3A mutational analysis in primary myelofibrosis, chronic myelomonocytic leukemia and advanced phases of myeloproliferative neoplasms," Leukemia. In press.

[68] R. D. Morin, N. A. Johnson, T. M. Severson et al., "Somatic mutations altering EZH2 (Tyr641) in follicular and diffuse large B-cell lymphomas of germinal-center origin," Nature Genetics, vol. 42, no. 2, pp. 181-185, 2010.

[69] O. Abdel-Wahab, A. Pardanani, J. Patel et al., "Concomitant analysis of EZH2 and ASXL1 mutations in myelofibrosis, chronic myelomonocytic leukemia and blast-phase myeloproliferative neoplasms," Leukemia. In press.

[70] T. Ernst, A. J. Chase, J. Score et al., "Inactivating mutations of the histone methyltransferase gene EZH2 in myeloid disorders," Nature Genetics, vol. 42, no. 8, pp. 722-726, 2010.

[71] G. Nikoloski, S. M. C. Langemeijer, R. P. Kuiper et al., "Somatic mutations of the histone methyltransferase gene EZH2 in myelodysplastic syndromes," Nature Genetics, vol. 42, no. 8, pp. 665-667, 2010.

[72] E. Viré, C. Brenner, R. Deplus et al., "The Polycomb group protein EZH2 directly controls DNA methylation," Nature, vol. 439, no. 7078, pp. 871-874, 2006.

[73] K. Döhner, J. Brown, U. Hehmann et al., "Molecular cytogenetic characterization of a critical region in bands 7q35-q36 commonly deleted in malignant myeloid disorders," Blood, vol. 92, no. 11, pp. 4031-4035, 1998.

[74] M. M. Le Beau, R. Espinosa III, E. M. Davis, J. D. Eisenbart, R. A. Larson, and E. D. Green, "Cytogenetic and molecular delineation of a region of chromosome 7 commonly deleted in malignant myeloid diseases," Blood, vol. 88, no. 6, pp. 1930-1935, 1996.

[75] G. Garcia-Manero, S. D. Gore, C. R. Cogle et al., "Evaluation of oral azacitidine using extended treatment schedules: a phase I study," Blood, vol. 116, 2010.

[76] A. S. Yang, K. D. Doshi, S. W. Choi et al., "DNA methylation changes after 5-aza-2'-deoxycytidine therapy in patients with leukemia," Cancer Research, vol. 66, no. 10, pp. 5495-5503, 2006.

[77] L. Zhou, X. Cheng, B. A. Connolly, M. J. Dickman, P. J. Hurd, and D. P. Hornby, "Zebularine: a novel DNA methylation inhibitor that forms a covalent complex with DNA methyltransferases," Journal of Molecular Biology, vol. 321, no. 4, pp. 591-599, 2002.

[78] J. C. Cheng, C. B. Matsen, F. A. Gonzales et al., "Inhibition of DNA methylation and reactivation of silenced genes by zebularine," Journal of the National Cancer Institute, vol. 95, no. 5, pp. 399-409, 2003.

[79] J. C. Cheng, C. B. Yoo, D. J. Weisenberger et al., "Preferential response of cancer cells to zebularine," Cancer Cell, vol. 6, no. 2, pp. 151-158, 2004.

[80] A. J. Davis, K. A. Gelmon, L. L. Siu et al., "Phase I and pharmacologic study of the human DNA methyltransferase antisense oligodeoxynucleotide MG98 given as a 21-day continuous infusion every 4 weeks," Investigational New Drugs, vol. 21, no. 1, pp. 85-97, 2003.

[81] D. J. Stewart, R. C. Donehower, E. A. Eisenhauer et al., "A phase I pharmacokinetic and pharmacodynamic study of the DNA methyltransferase 1 inhibitor MG98 administered twice weekly," Annals of Oncology, vol. 14, no. 5, pp. 766-774, 2003.

[82] R. B. Klisovic, W. Stock, S. Cataland et al., "A phase I biological study of MG98, an oligodeoxynucleotide antisense to DNA methyltransferase 1, in patients with high-risk myelodysplasia and acute myeloid leukemia," Clinical Cancer Research, vol. 14, no. 8, pp. 2444-2449, 2008.

[83] E. Winquist, J. Knox, J. P. Ayoub et al., "Phase II trial of DNA methyltransferase 1 inhibition with the antisense oligonucleotide MG98 in patients with metastatic renal carcinoma: a National Cancer Institute of Canada Clinical Trials Group investigational new drug study," Investigational New Drugs, vol. 24, no. 2, pp. 159-167, 2006.

[84] B. Brueckner, R. G. Boy, P. Siedlecki et al., "Epigenetic reactivation of tumor suppressor genes by a novel smallmolecule inhibitor of human DNA methyltransferases," Cancer Research, vol. 65, no. 14, pp. 6305-6311, 2005.

[85] D. Kuck, N. Singh, F. Lyko, and J. L. Medina-Franco, "Novel and selective DNA methyltransferase inhibitors: dockingbased virtual screening and experimental evaluation," Bioorganic and Medicinal Chemistry, vol. 18, no. 2, pp. 822-829, 2010.

[86] R. Itzykson, O. Kosmider, T. Cluzeau et al., "Impact of TET2 mutations on response rate to azacitidine in myelodysplastic syndromes and low blast count acute myeloid leukemias," Leukemia. In press.

[87] D. A. Pollyea, A. Raval, B. Kusler, J. R. Gotlib, A. A. Alizadeh, and B. S. Mitchell, "Impact of TET2 mutations on mRNA expression and clinical outcomes in MDS patients treated with DNA methyltransferase inhibitors," Journal of Hematology \& Oncology, 2010.

[88] A. V. Krivtsov and S. A. Armstrong, "MLL translocations, histone modifications and leukaemia stem-cell development," Nature Reviews Cancer, vol. 7, no. 11, pp. 823-833, 2007.

[89] G. G. Wang, L. Cai, M. P. Pasillas, and M. P. Kamps, "NUP98NSD1 links H3K36 methylation to Hox-A gene activation and leukaemogenesis," Nature Cell Biology, vol. 9, no. 7, pp. 804-812, 2007.

[90] C. Meyer, E. Kowarz, J. Hofmann et al., "New insights to the MLL recombinome of acute leukemias," Leukemia, vol. 23, no. 8, pp. 1490-1499, 2009. 
[91] S. Y. Jo, E. M. Granowicz, I. Maillard, D. Thomas, and J. L. Hess, "Requirement for Dot1l in murine postnatal hematopoiesis and leukemogenesis by MLL translocation," Blood, vol. 117, no. 18, pp. 4759-4768, 2011.

[92] K. Bernt, N. Zhu, J. Faber et al., "Demonstration of a role for Dot1l in MLL-rearranged leukemia using a conditional loss of function model," Blood, vol. 116, 2010.

[93] R. Pollock, S. R. Daigle, E. J. Olhava et al., "Selective killing of mixed lineage leukemia cells by a potent small-molecule DOT1L inhibitor," Blood, vol. 116, 2010.

[94] A. V. Krivtsov, Z. Feng, M. E. Lemieux et al., "H3K79 methylation profiles define murine and human MLL-AF4 leukemias," Cancer Cell, vol. 14, no. 5, pp. 355-368, 2008.

[95] A. Bursen, K. Schwabe, B. Rüster et al., "The AF4·MLL fusion protein is capable of inducing ALL in mice without requirement of MLL·AF4," Blood, vol. 115, no. 17, pp. 35703579, 2010.

[96] A. Benedikt, S. Baltruschat, B. Scholz et al., "The leukemogenic AF4-MLL fusion protein causes P-TEFb kinase activation and altered epigenetic signatures," Leukemia, vol. 25, pp. 135-144, 2010.

[97] A. T. Nguyen, B. Xiao, R. L. Neppl et al., "DOT1L regulates dystrophin expression and is critical for cardiac function," Genes and Development, vol. 25, no. 3, pp. 263-274, 2011.

[98] A. Jankowska, H. Makishima, R. V. Tiu et al., "Mutational spectrum in chronic myelomonocytic leukemia includes genes associated with epigenetic regulation such as UTX and EZH2," Blood, vol. 116, 2010.

[99] M. Allan, S. Manku, E. Therrien et al., "N-benzyl-1-heteroaryl3-(trifluoromethyl)-1H-pyrazole-5-carboxamides as inhibitors of co-activator associated arginine methyltransferase 1 (CARM1)," Bioorganic and Medicinal Chemistry Letters, vol. 19, no. 4, pp. 1218-1223, 2009.

[100] J. Eeckhoute, E. K. Keeton, M. Lupien, S. A. Krum, J. S. Carroll, and M. Brown, "Positive cross-regulatory loop ties GATA-3 to estrogen receptor $\alpha$ expression in breast cancer," Cancer Research, vol. 67, no. 13, pp. 6477-6483, 2007.

[101] Y. R. Kim, B. K. Lee, R. Y. Park et al., "Differential CARM1 expression in prostate and colorectal cancers," BioMed Central Cancer, vol. 10, p. 197, 2010. 


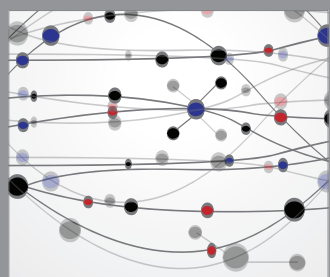

The Scientific World Journal
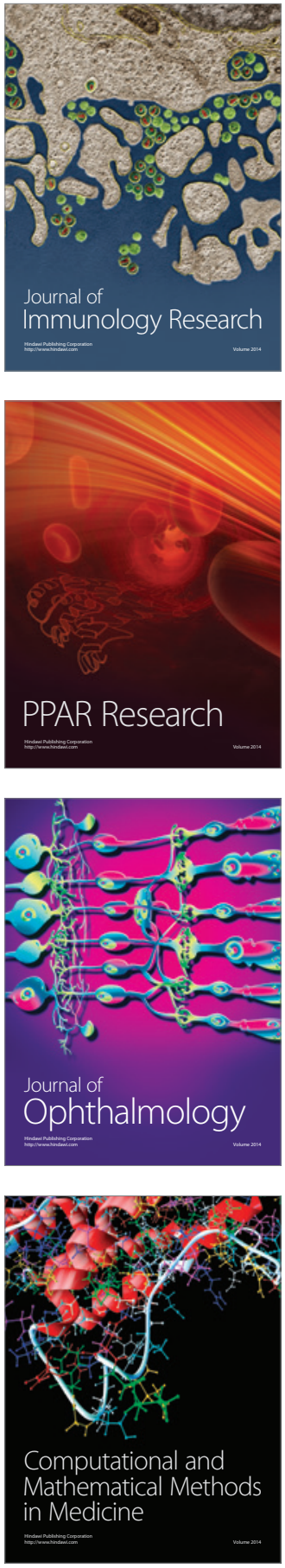

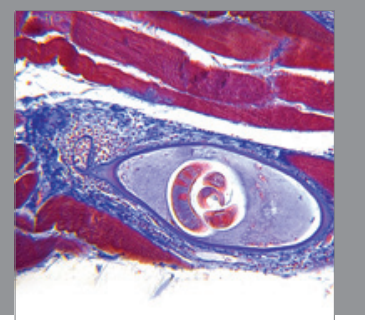

Gastroenterology

Research and Practice
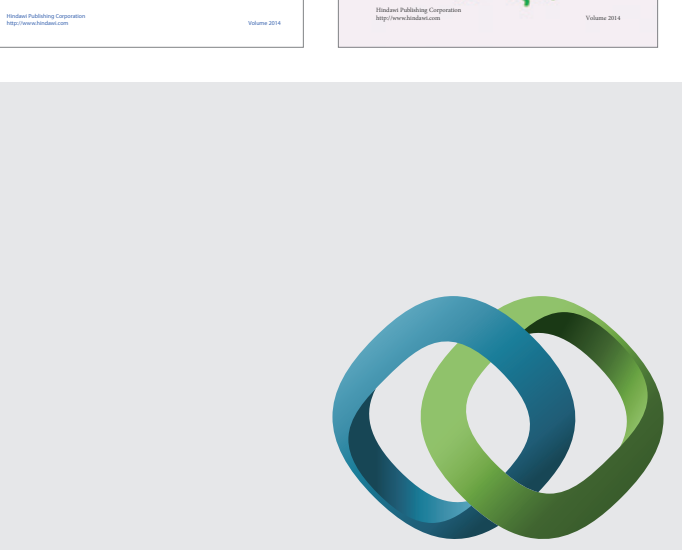

\section{Hindawi}

Submit your manuscripts at

http://www.hindawi.com
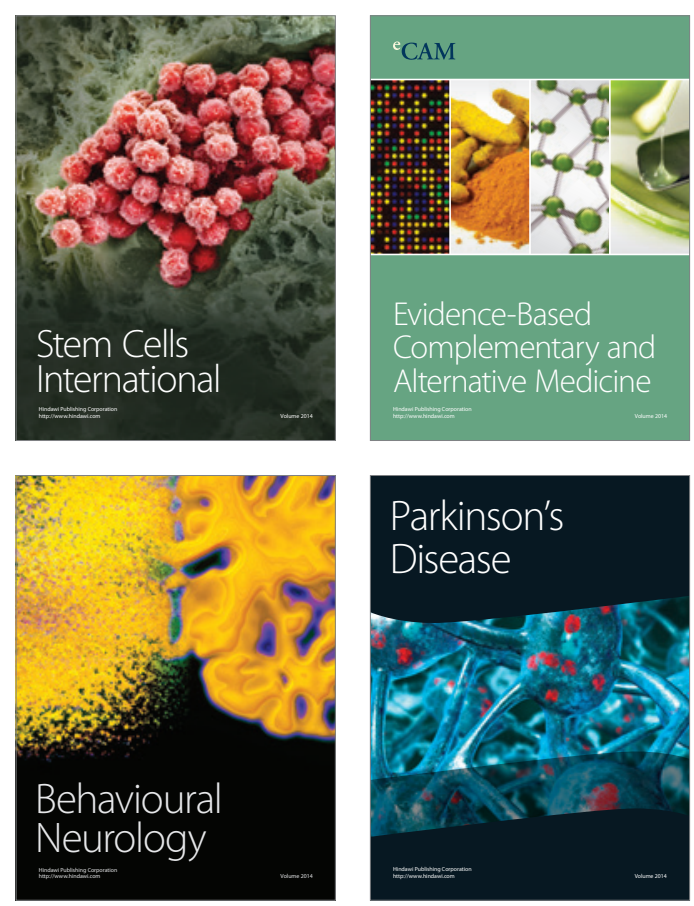

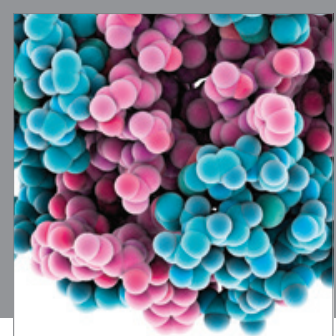

Journal of
Diabetes Research

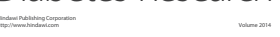

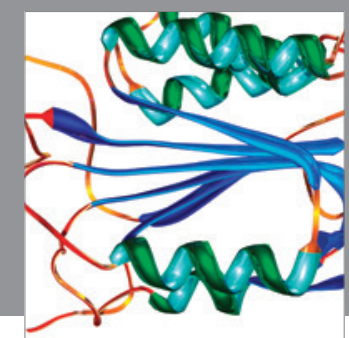

Disease Markers
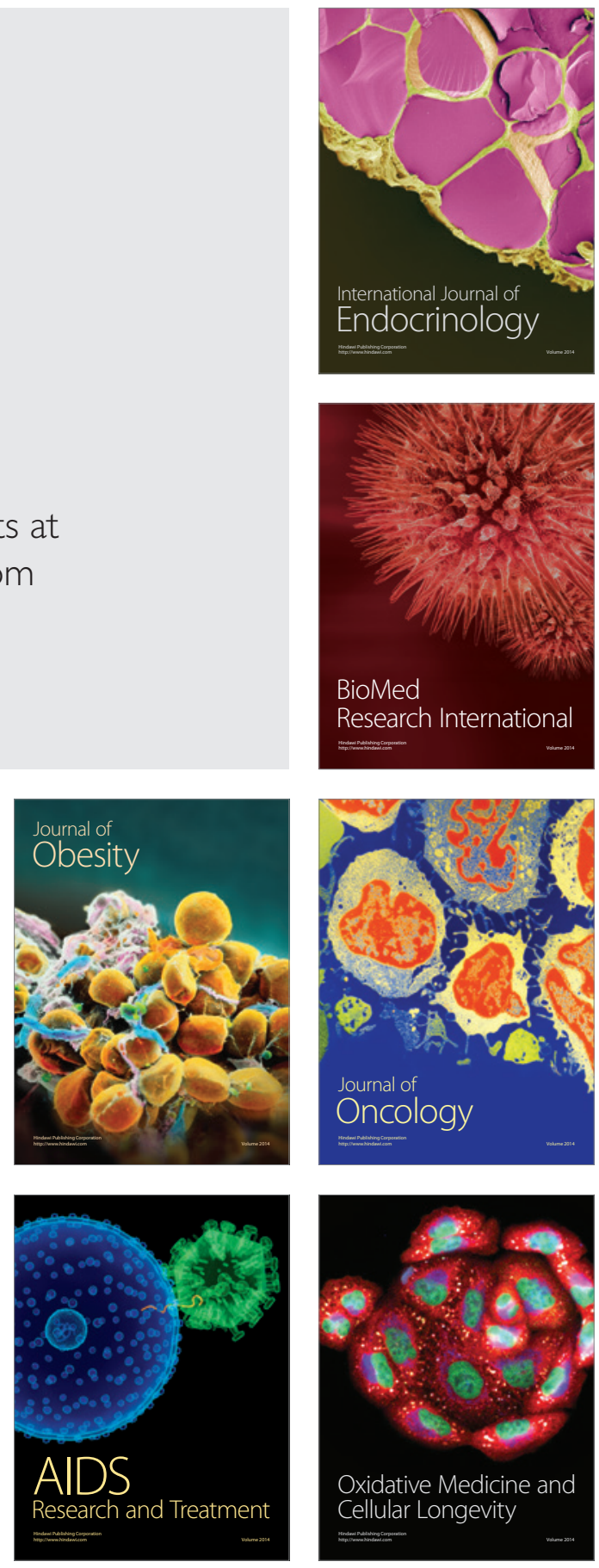